\title{
Una académica comprometida con el estudio de la historia del pensamiento iuspolítico alemán y el trabajo de Hans Kelsen: una aproximación a la apasionante obra de Sara Lagi*
}

Entrevista realizada a Sara Lagi por:

\section{Augusto Fernando Carrillo Salgado**}

Universidad Nacional Autónoma de México (Ciudad de México, México)

\footnotetext{
* Entrevista traducida del italiano al castellano por Augusto Fernando Carrillo Salgado bajo la supervisión de Sara Lagi.

** Licenciado y maestro en Derecho, con mención honorífica, por la Universidad Nacional Autónoma de México (México); especialista como secretario de juzgado (curso básico de secretarios) por la Escuela Judicial del Instituto de la Judicatura Federal (hoy Escuela Federal de Formación Judicial). Correo electrónico: fernando90@comunidad.unam.mx ORCID: https://orcid.org/00000001-6107-4917
} 
DOI:

https://doi.org/10.18046/prec.v19.4650 


\section{Semblanza}

Sara Lagi obtuvo el grado de doctora en Historia del Pensamiento Político Europeo por la Universidad de Perugia en el año 2005. Actualmente es catedrática de la Universidad de Turín; también ha sido docente en la Universidad de Florencia (2007-2009), Middlebury College, Smith College y Fashion Institute of Technology (2005-2014). A lo largo de su prolífica trayectoria docente, Sara Lagi ha publicado un gran número de artículos y libros, entre los que destacan: "Pensare la democracia: Hans Kelsen e Hermann Heller a confronto"; "Kelsen e la Corte costituzionale austriaca: un percorso storico-politico (1918-1920)"; "Hans Kelsen, un pensatore democrático tra Europa e America (1920-1955)"; Il Pensiero político di Hans Kelsen (1911-1920). Le origini di Essenza e valore della democracia; "Georg Jellinek storico del pensiero politico (18831905)"; "Adolf Fischer e Karl Renner: la questione nazionale austriaca (1869-1917)"; "The formation of a liberal thinker: Georg Jellinek and his early writings"; "Hans Kelsen and the Austrian Constitutional Court (1918-1929)"; "Karl Renner: Staat und Nation"; "Hans Kelsen: pensador político"; “Territorio y pueblo en Hans Kelsen”.

Palabras claves: Historia del pensamiento iusfilosófico alemán; Hans Kelsen; Georg Jellinek; República de Weimar.

\section{Semblance}

Sara Lagi got her $\mathrm{PhD}$ in History of European Political Thought by University of Perugia in 2005. Currently, she is Associate Professor at University of Turin; however, she taught before at University of Florence (2007-2009), Middlebury College, Smith College and Fashion Institute of Technology (2005-2014). She has published, through her prolific career, a huge number of important books and papers such as: Pensare la democracia: Hans Kelsen e Hermann Heller a confronto; Kelsen e la Corte costituzionale austriaca: un percorso storico-politico (1918-1920); Hans Kelsen, un pensatore democrático tra Europa e America (1920-1955); Il Pensiero político di Hans Kelsen (1911-1920). Le origini di Essenza e valore della democracia; Georg Jellinek storico del pensiero politico (1883-1905); Adolf Fischer e Karl Renner: la questione nazionale austriaca (1869-1917); The formation of a liberal thinker: Georg Jellinek and his early writings; Hans Kelsen and the Austrian Constitutional Court (1918-1929); Karl Renner: Staat und Nation; Hans Kelsen: pensador político; Territorio y pueblo en Hans Kelsen.

Keywords: History of German philosophical thought; Hans Kelsen; Georg Jellinek; Weimar Republic. 

Augusto Fernando Carrillo Salgado (AFCS): Ante todo, estimada profesora, le agradezco el tiempo que generosamente me dispensa; máxime al tomar en consideración el gran número de responsabilidades académicas que una intelectual de su talla tiene que atender cotidianamente. Creo no equivocarme al afirmar lo siguiente: aun confinados en nuestras moradas, pareciera ser que los días transcurren con mayor rapidez. Así, el tiempo es, sin el menor atisbo de dudas, uno de los regalos más preciosos que un ser humano puede dispensar a otro.

A lo largo de su prolífica trayectoria académica ha estudiado, con sobrada maestría, diversos temas. Desde luego, unas cuantas líneas no podrían dar cuenta exhaustiva de todos y cada uno de estos. Por tanto, desearía conversar sobre un par de asuntos en particular. Verbigracia, su apasionado interés por el estudio de la historia del pensamiento iuspolítico alemán de finales del siglo XIX y principios del XX, así como sobre la vida y obra de Hans Kelsen.

Sin embargo, previamente me gustaría formularle unas cuantas preguntas acerca de su trayectoria académica. Estoy convencido de que la experiencia vital del ser humano y su obra se encuentran, indefectiblemente, vinculados. En otras palabras, la producción académica de las personas es el reflejo de sus vivencias, inquietudes e intereses. En tal virtud, ipodría explicarnos, estimada profesora, cómo nace su pasión por la historia del derecho?, iqué profesores influyeron de manera decisiva en su formación intelectual?

Sara Lagi (SL): Antes que nada, le agradezco por sus palabras tan gentiles y generosas hacia mi persona, las cuales me honran. A decir verdad, soy pésima para hablar de mí misma; sencillamente me resulta difícil, pero trataré. Mi formación no es jurídica; aquello que usted llama "historia del derecho" prefiero denominarlo, de manera más directa, historia del pensamiento iuspolítico. Este es el ámbito principal de mi interés científico, es decir, la conexión entre la reflexión política y jurídica en relación con temas como el Estado, la libertad, el vínculo entre el Estado y los ciudadanos, los derechos fundamentales. De manera precisa, a través de la obra de pensadores que, para mí, han encarnado el encuentro entre estas dos dimensiones del pensamiento, como Hans Kelsen, Georg Jellinek, Hermann Heller, por citar algunos autores de los cuales me he ocupado, pero, obviamente, sin perder de vista a muchos otros. Hablar de una reflexión iuspolítica no significa ignorar o desconocer las diferencias metodológicas y las peculiaridades en términos de contenido y exigencias de investigación que caracterizan al pensamiento jurídico y político, sino darse 
cuenta de que, debido a sus diferencias, estas dos tradiciones del pensamiento dialogan entre ellas, porque, al final, tratan temas comunes como el poder, la libertad, el humano en la sociedad e historia. Es, precisamente, este diálogo lo que me ha fascinado y continúa interesándome. A esto me he acercado y sigo aproximándome, aunque con gran cautela, porque mi formación es principalmente histórico-política, más que jurídica.

Por lo que respecta a mi formación académica, me gradué en Florencia con una tesis en historia de la doctrina política, dedicada a la teoría democrática de Hans Kelsen, bajo la supervisión de la profesora Lea Campos Boralevi, catedrática de historia de las doctrinas políticas, cuyas enseñanzas han sido fundamentales para mí. Me enseñó que el problema no es elaborar una investigación que se encuentre exenta de críticas, porque solo aquello que es estéril no suscita objeciones de ningún tipo. Hoy, todavía, estoy personalmente muy agradecida hacia quien me ayuda, con críticas constructivas, a profundizar en mis temas de estudio. Estoy convencida de que hacer investigación significa confrontarse, no tanto con quien está perfectamente de acuerdo con nuestras convicciones, sino, sobre todo, con quien no lo está, porque es justamente en este modo que se puede ver un problema o una cuestión desde un punto de vista diferente. Otra figura importante para mí ha sido la del profesor Arduino Agnelli, catedrático de historia del pensamiento político de la Universidad de Trieste, finado hace muchos años, prematuramente. Me orientó en las primeras etapas de la elaboración de mi tesis de doctorado, alentándome a estudiar el pensamiento político de Kelsen desde un punto de vista histórico.

AFCS: Muchas gracias, profesora, por narrarnos estos personales e interesantes pasajes de su vida. No quisiera desaprovechar la ocasión, por otra parte, para expresar mi profundo respeto y admiración hacia su trabajo. Como es ampliamente conocido, ha estudiado la historia del pensamiento iuspolítico alemán de finales del siglo XIX y principios del XX con sobrado rigor metodológico y precisión conceptual. iNo me equivoco al afirmar que su obra es lo mejor que se ha escrito al respecto en lengua italiana en las últimas décadas! ¿Cómo nace ese interés?

SL: Dicho interés está ligado propiamente a la obra de Kelsen. Afrontar su teoría democrática y, obviamente, medirme con su teoría jurídica me ha "constreñido" a analizar la tradición del pensamiento iuspolítico en lengua germana entre las postrimerías del siglo XIX y la primera mitad del XX. No podría, de hecho, 
pretender estudiar su pensamiento político sin indagar su relación con la traditionelle Staats y Rechtslehre alemana de Jellinek, por ejemplo, o su deuda hacia el neokantismo o, más aún, su posición de jurista e intelectual al interior de aquél gran laboratorio jurídico y político que fue Weimar. Es un interés, por tanto, que nace primeramente de una exigencia de comprensión y contextualización y, después, como muchas veces ocurre, ha devenido en un objeto de estudio por derecho propio.

AFCS: A lo largo de su trayectoria académica ha dedicado un impresionante número de páginas al estudio de Hans Kelsen. Tal y como ha precisado, tanto su tesis de licenciatura como la de doctorado versaron sobre la vida y obra del jurista austriaco. Más tarde, publicó un libro que hoy resulta de lectura obligatoria para la comprensión del pensamiento iuspolítico kelseniano; a saber: Il Pensiero político di Hans Kelsen (1911-1920). Le origini di Essenza e valore della democrazia.

Sin duda alguna, usted ha mostrado al jurista desde una perspectiva dinámica, como un destacado contribuyente en la confección de la constitución de Austria, a lado de Karl Renner, como juez del primer tribunal constitucional austriaco y como un brillante contendiente en los intensos debates teórico-políticos durante la República de Weimar.

Personalmente, considero que desde hace varias décadas, en Latinoamérica, Kelsen ha sido analizado tan solo desde su dimensión metodológica, o perspectiva estática, la cual se encuentra reflejada esencialmente en su Reine Rechtslehre. Empero, usted lo muestra desde un ángulo más humano, como un jurista comprometido con sus ideales. Estoy convencido de que esa frescura sobre la vida y obra del jurista se debe a su aproximación con la historiografía, así como su distanciamiento de los métodos estrictamente jurídicos. ¿En qué momento brota este interés por estudiar la vida y obra de Hans Kelsen?

SL: Como creo que se desprende, en parte, de mis respuestas anteriores, el interés por la obra de Kelsen se remonta a los años en la Universidad de Florencia. Me topé con este pensador por primera vez leyendo Storia della democrazia in Europa de Salvo Mastellone. Campos Boralevi se sorprendió cuando le propuse una tesis de licenciatura sobre Kelsen, sea porque era un autor del todo ignorado en la facultad de Historia que frecuentaba, sea porque era un jurista; sin embargo, aceptó el reto de seguirme por ese largo camino. ¿Por qué Kelsen? Me llamaron la atención dos aspectos. En primer término, el hecho de que, tras la Gran 
Guerra, él hubiera sido uno de los poquísimos intelectuales que defendieron a capa y espada la democracia liberal, el parlamentarismo y el pluralismo político, denunciando bien los movimientos antisistema de extrema derecha, bien aquellos de izquierda radical. En suma, me llamó la atención el hecho de que, en un periodo histórico caracterizado, en gran parte del continente, por la propagación de los extremismos -ideologías que predicaban la realización de una sociedad perfecta, siempre y cuando fuera, obviamente, eliminada toda forma de disenso-, hubiera un intelectual que, de manera completamente contracorriente, reivindicara una visión moderada y racional de la convivencia política y civil. En segundo lugar, leyendo la literatura en aquel entonces vigente, me pareció que las etiquetas de "formalista" y "procedimentalista" atribuidas a Kelsen eran, en cierto modo, reduccionistas. En cuanto historiadora, pensé que podría ser interesante tratar de contextualizar a Kelsen como pensador político, tratar de comprender si existió y cuál fue la conexión entre su visión política y la realidad histórico-política en la que vivió y trabajó. Al final, esto ha sido el objetivo principal de mi tesis de doctorado sobre la génesis de la primera edición de Vom Wesen und Wert der Demokratie.

AFCS: Hans Kelsen fue hombre de luenga vida (1881-1973). iSu existencia casi alcanzó los cien años! El jurista fue un único ser, además de un ser único, que atravesó diferentes facetas. Es decir, podría afirmarse, hasta cierto punto, que existieron uno y varios Hans Kelsen: el académico de la Universidad de Viena, el arquitecto de la Constitución austriaca, el juez del Tribunal Constitucional de Austria, el maestro de Alemania y Praga, así como el jurista exiliado en los Estados Unidos de América. La obra de Hans Kelsen, al igual que su propia existencia, estuvo en un proceso de refinamiento constante. En efecto, muchos de sus pensamientos fueron matizados y desarrollados, en tanto que otros fueron desechados por completo. En mi opinión, el desarrollo del pensamiento kelseniano puede observarse nítidamente en tres de sus obras: su Hauptprobleme der Staatsrechtslehre, su Reine Rechtslehre (ambas versiones) y la póstuma Allgemeine Theorie der Normen. ¿Podría indicarnos qué elementos, a pesar de la mutación del pensamiento kelseniano, se encuentran presentes en toda la obra de Hans Kelsen?

SL: Es una pregunta compleja. Trataré de responderla por conceptos clave. Desde un punto de vista de la teoría jurídica, advertimos, en mi opinión, una 
mayor apertura por parte de Kelsen. Le pongo un ejemplo concreto: la General Theory of Law and State (1945) -que, como recordaba un gran estudioso italiano de Kelsen, Renato Treves, puede ser considerada la versión americana de la Reine Rechtslehre-admitió de manera muy informal la existencia de una pluralidad de ciencias del derecho, entre ellas la sociología del derecho, que había sido objeto de un ataque frontal por parte de Kelsen durante la década de 1910. En aquella época, Kelsen se encontraba en una larga y animada disputa con Eugen Ehrlich, uno de los pioneros de la sociología del derecho, sobre la relación entre la ciencia jurídica y las ciencias sociales. Kelsen fue, entonces, muy claro al defender la separación entre estos dos ámbitos del saber, bajo la luz del proceso de purificación de la ciencia jurídica que emprendió en su Hauptprobleme der Staatsrechtslehre (1911). Por si fuera poco, en Europa, Kelsen demostró un profundo interés por el tema de la justicia constitucional. Basta solo pensar en el largo ensayo en francés La garantie juridictionelle de la constitution (1928), en el que vinculó la justicia constitucional y la defensa de las minorías. Concretamente, también dio una contribución fundamental a la creación de la Corte Constitucional Austriaca, introduciendo el concepto de Verfassungsgerichtshof como "defensor objetivo de la constitución". Además, el papel y significado de la Corte Constitucional fue el centro de su célebre desacuerdo con Carl Schmitt en Der Hüter der Verfassung (1931). Aquí, en mi opinión, este Kelsen constitucionalista casi desapareció del todo en América, para dar paso a un Kelsen internacionalista. Un cambio que, como ha subrayado oportunamente la literatura reciente, respondía también a la exigencia personal y profesional de adaptarse a un ambiente académico muy diverso de aquél del que provenía. En el plano de la teoría democrática, estoy también convencida de que no hay una profunda ruptura entre el Kelsen europeo y el estadunidense: cambia el contexto histórico-político, cambian las exigencias, los objetivos (al menos en parte), pero las ideas, los principios permanecen intactos. También en su Foundations of Democracy (1955) encontramos una concepción liberal, relativista, pluralista, procedimentalista de la democracia.

AFCS: Salvo su mejor consideración, usted ha analizado, esencialmente, dos periodos de la vida de Kelsen. El primero corresponde a su participación en la confección de la constitución austriaca, así como su faceta en cuanto juez del Tribunal Constitucional de Austria. El segundo es el relativo al jurista de los acalorados debates de la República de Weimar y el Kelsen que parte al exilio en los Estados Unidos de América. El primer periodo está perfectamente descrito en 
su libro Il pensiero politico di Hans Kelsen (1911-1920), así como en sus artículos "Hans Kelsen and the Austrian Constitutional Court (1918-1929)" y "Kelsen e la Corte costituzionale austriaca: un percorso storico-politico (1918-1920)". La segunda etapa ha sido abordada en "Hans Kelsen un pensatore democratico tra Europa e America (1920-1955)”. ¿Podría pintarnos un retrato del Hans Kelsen del primer periodo? ¿Cuáles son las motivaciones, intereses y preocupaciones de este joven Hans Kelsen?

SL: Es un joven Kelsen que debutó en 1905 con un largo ensayo dedicado a la Staatslehre des Dante Alighieri, en el cual demuestra un elevado conocimiento de la historia del pensamiento jurídico medieval. Es interesante, en mi opinión, recordar cómo el teórico de la Teoría Pura del Derecho se aparece ante el mundo científico y académico de la época con un escrito relacionado con la historia del derecho y en el que, además, lee la concepción dantesca del Imperio (monarquía), proyectando sobre ella una visión iuspolítica sustancialmente moderna y, en ciertos aspectos, que recuerda a las lecciones de Otto Von Gierke. Kelsen habla, de hecho, sobre la teoría dantesca del Estado, haciendo así una operación históricamente muy cuestionable; es decir, retrasando demasiado la aparición del concepto mismo del Estado. Sin embargo, en mi opinión, detrás del interés por el concepto dantesco de un imperio pacificador grande y único, se puede comenzar a entrever aquella exigencia por pensar en términos monistas que, después, se sustanciará en sus sucesivas obras teórico-jurídicas.

Está, además, el joven Kelsen de Hauptprobleme der Staatsrechtslehre, que rompe de manera muy brusca con la tradición jurídica que le precede, colocando radicalmente en discusión el significado de la ciencia jurídica, despersonalizando el concepto del Estado, que es identificado con el ordenamiento jurídico y llevando al extremo el principio mismo del Estado de derecho. No imaginaba un Estado limitado por el derecho, sino, más bien, un Estado que coincide con el derecho mismo. Y en esta compleja operación, que le granjea no pocas críticas, Kelsen no tiene tan solo una importante deuda con el neokantismo de Cohen, sino también -como él mismo reconocerá en 1922- hacia Sigmund Freud, de quien aprende el vínculo entre la personificación del Estado, de la sociedad y Dios con la "psicología individual". Es un Kelsen, en ciertos aspectos, de ruptura, que busca dejar una impronta original y personal en la reflexión jurídica, que busca su propia voz y su propio camino. Además, es precisamente a partir de Hauptprobleme que elabora una definición de parlamento, como órgano 
intrínsecamente plural, del cual no es posible prescindir para comprender, después, el desarrollo de su pensamiento político y democrático. Además, me gustaría recordar que el joven Kelsen demostraba también un vivo interés por la política en su dimensión más concreta: en 1907 publica el ensayo Wählerlisten und Reklamationsrecht, con el cual interviene en favor del derecho electoral del Imperio austrohúngaro, en un año crucial para la historia de los Habsburgo. Es, de hecho, en 1907 cuando viene introducido el voto directo universal masculino.

AFCS: iNo podría estar más de acuerdo con usted! En la obra kelseniana del primer periodo pueden apreciarse nítidamente las improntas de Immanuel Kant y Georg Jellinek. La presencia de las ideas kantianas plasmadas en Kritik der reinen Vernunft y Zum Ewigen Frieden es clara en Hauptprobleme der Staatsrechtslehre, Die Idee des Naturrechts y en la primera versión de la Reine Rechtslehre. Por razones obvias, Kelsen no tuvo la oportunidad de convivir directamente con el maestro de Köningsberg. Sin embargo, con quien sí tuvo un trato directo, como usted explica en Hans Kelsen and the Austrian Constitutional Court (1918-1929), es con Georg Jellinek.

Sobre Georg Jellinek usted también ha escrito bastante; verbigracia, "Hans Kelsen e Georg Jellinek: il parlamento" (capítulo II de Il pensiero politico di Hans Kelsen), "The Formation of a Liberal Thinker: Georg Jellinek and his Early Writings (1872-1878)" y "Georg Jellinek, a Liberal Political Thinker against Despotic Rule (1885-1898)”. En mi opinión, empero, los vínculos teóricos y personales entre Hans Kelsen y Georg Jellinek no han sido suficientemente explorados en la investigación de habla hispana. ¿Podría explicarnos cual fue la relación entre el jurista austriaco y el maestro Jellinek? ¿En qué momento se conocen? ¿De qué manera influyó Georg Jellinek sobre la obra de Hans Kelsen? ¿Piensa continuar escribiendo sobre esta relación en lo sucesivo?

SL: Es el propio Kelsen quien cuenta, por así decirlo, de manera directa, su relación con Jellinek. En el prefacio de la primera edición de Hauptprobleme (1911) recordaba a Jellinek como un docente estudioso, como un punto de referencia.

Mi impresión es que el homenaje a Jellinek fue, en cierto modo, funcional para subrayar el alcance de la ruptura científica imbuida en su impresionante monografía de 1911. Kelsen reconoció a Jellinek la talla de un gran estudioso, para enfatizar con mayor eficacia el carácter revolucionario, original e innovador de su teoría jurídica y de su particular visión de la ciencia jurídica. Al menos, 
eso es lo que yo pienso. Al mismo tiempo -como prueba de lo difícil que es encasillar la relación entre ambos- cabe recordar aquello que Kelsen escribió sobre Jellinek en sus memorias autobiográficas. De aquellas aprendemos que Kelsen conoció en persona a Jellinek en Heidelberg. Fue Edmund Bernatzik, un importante constitucionalista y docente de derecho público en Viena, quien aconsejó al joven y prometedor alumno que asistiera a las lecciones de Jellinek. Este consejo no pudo ser más erróneo. Entre ambos -de acuerdo con Kelsenhubo una antipatía a primera vista. Simplemente se encontraban insoportables el uno al otro. De manera particular, Kelsen acusó a Jellinek de ser un docente vanidoso y habituado a rodearse por aduladores. Kelsen reconoció en Jellinek un gran historiador del derecho, pero, ya en tiempos de Heidelberg, tenía muchas dudas sobre la solidez científica de su concepción dual del Estado, que, de hecho, después fue duramente criticada propiamente en Hauptprobleme. Francamente, el juicio de Kelsen sobre Jellinek siempre me ha parecido un poco severo. Pero los disgustos pueden ser, en ocasiones, verdaderamente feroces. Por lo que a mi respecta, me gustaría tener en el futuro la oportunidad de intentar nuevamente las traducciones de los Schriften und Reden de Jellinek, continuando así un trabajo iniciado entre 2008 y 2009, cuando publiqué la traducción de algunos de sus Schriften dedicados a la historia de las ideas jurídicas y políticas. Quizá.

AFCS: Francamente, me encuentro sorprendido por su erudición. Sobre todo, estoy anonadado por la sencillez con la que explica una relación tan compleja como aquella entre Hans Kelsen y Georg Jellinek. A decir verdad, nunca me habría imaginado que la relación entre ambos genios hubiese sido tan ríspida. Aunque con notables diferencias, el control de la constitucionalidad kelseniano no podría negar en forma alguna sus raíces en los postulados de Jellinek. En cierto sentido, uno podría creer que de Kelsen hacia Jellinek habría existido un vínculo de admiración, un lazo fraterno. Sin embargo, fue todo lo contrario. Lo cual no deja de ser, por supuesto, muy interesante y revelador, en cuanto subraya la honestidad científica de Kelsen, quien, no obstante los defectos observados en Jellinek, jamás negó sus aportaciones a la ciencia jurídica. Creo que Hans Kelsen supo separar muy bien sus antipatías personales de su valoración objetiva respecto a la obra de Jellinek.

Ciertamente, tanto la impronta de Jellinek como la propia de Hans Kelsen tuvieron un significado capital en el periodo histórico denominado República de Weimar. Esta época (1919-1933) es, sin el menor atisbo de duda, uno de 
los periodos de la historia alemana más apasionantes que puede haber, porque es un tiempo de radicales claroscuros. Por una parte, se caracterizó por la inestabilidad económica, así como por una crisis política que estremeció los cimientos de todas las instituciones de Alemania. Por otro lado, también fue un periodo de grandes ideas. iLa República de Weimar fue una época de propuestas intelectuales innovadoras! iFue el escenario de los geniales y apasionantes debates entre Carl Schmitt, Hermann Heller, Rudolf Smend y Hans Kelsen! Debido a esta última característica, algunos académicos la han denominado también "Laboratorio de Weimar".

Por supuesto, usted ha escrito documentos interesantísimos sobre este periodo. En su artículo "Pensare la democrazia: Hans Kelsen e Hermann Heller", por ejemplo, ha explicado el apasionante debate entre ambos juristas en torno a la forma en que la democracia debe entenderse. Mientras aquel busca la armonía social por medio de un sistema normativo caracterizado por una coherencia lógica cuasi perfecta, este trata de calmar los ánimos de la turbulenta sociedad alemana por medio del debate y acuerdos parlamentarios. Cabe destacar que la discusión entre ambos juristas alcanzó tal acaloramiento que Hermann Heller llegó a reclamarle a Hans Kelsen la desconexión de su propuesta con la realidad; inclusive, calificó las ideas kelsenianas como una suerte de teoría del derecho aburguesada. Aunque en principio ambas posturas lucen casi incompatibles, no dejan de compartir un elemento común: su base neokantiana. Tanto la propuesta de Hermann Heller como la de Hans Kelsen son un llamado a la paz pero por vías diferentes, quizá opuestas. No lo sé...

El debate Heller-Kelsen, así como una parte de la República de Weimar tienen lugar en el marco del primer periodo de la obra kelseniana (1911-1920). ¿Podría explicarnos el contexto de la República de Weimar? ¿Cuándo y por qué surge? ¿Cuáles fueron sus características? Además, ipodría presentar, grosso modo, el debate entre Hermann Heller y Hans Kelsen que analiza en su artículo?

SL: Weimar fue un extraordinario laboratorio político. La trayectoria de la república fue realmente breve, de 1919 a 1933, y al mismo tiempo extremadamente intensa. Uno de los términos que más se repiten para describir la efímera vida de la República weimariana es "crisis". Tal vez, gracias a ella se liberaron grandes energías intelectuales. Entre los años veinte y treinte, pensadores como Carl Schmitt, Hermann Heller, Ernst Fraenkel, Gerhard Leibholz, Rudolf Smend y el propio Kelsen, que se había mudado a Colonia en 1930, reflexionaron, con sus 
particularidades, sobre temas cruciales, como el significado de soberanía, Estado, representación política y pluralidad social, no por amor hacia las abstracciones, sino para descifrar y posiblemente resolver la crisis que aquejaba a la joven república alemana. Esta se fundaba sobre una constitución democrática, muy progresista, que constitucionalizaba los derechos sociales, y que, sin embargo, en breve tiempo había demostrado ser frágil e incierta, presa de una crítica falta de estabilidad. A los ojos de aquella parte de la sociedad germana que había aceptado a regañadientes el fin del imperio, la república parecía ser una elección desafortunada. En particular, el pluralismo partidario que, en cualquier modo, era enfatizado por un sistema de representación de tipo proporcional era considerado por las fuerzas y personalidades conservadoras un factor de caos y ruptura de la unidad política.

En suma, este era el contexto en que se situaban Heller y Kelsen. Desde el punto de vista de la teoría jurídica, los dos estaban, sin duda alguna, en desacuerdo. El célebre ensayo helleriano sobre la Souveränität (1927) fue un duro reproche al formalismo jurídico kelseniano por parte de Heller, considerado el resultado más extremo y peligroso de aquél complejo proceso de despersonalización del concepto de Estado y soberanía que conducía al corazón mismo de la modernidad europea y occidental. Y, sin embargo, creo que todavía hay más. En el plano del pensamiento político hay interesantes puntos de contacto entre los dos que he tratado de examinar, pero que merecen un estudio ulterior más profundo. Ambos estaban a favor del pluralismo político y partidario -a diferencia de Schmitt, por ejemplo- y ambos consideraban central la garantía de las libertades fundamentales, se declaraban en favor del sistema proporcional y, sobre todo, 220 pensaban que la unidad política no era una suerte de realidad preconstituida, a la que se le daba un voto para todos, sino algo que surgía esencialmente de un proceso de "integración" de la pluralidad social y política. Ciertamente, Heller -un jurista socialdemócrata- creía, a diferencia de Kelsen, que esta "integración" debía conducir a la clase obrera a desempeñar un papel político y social de gran envergadura que, hasta entonces, no había tenido, pero, más allá de esto, considero que sería útil volver a estudiar a Heller y Kelsen en relación con sus puntos de contacto. Se podría así-al menos eso creo-leer la experiencia weimariana, superando un poco los rígidos parapetos entre Kelsen, por un lado, y Heller, por el otro. Es esta su visión común del pluralismo, entendido como un factor positivo -si bien integrado- que debería reexaminarse en relación con el debate iuspolítico weimeriano. 
AFCS: Como se señaló con anterioridad, usted identifica una segunda etapa en la vida y obra del jurista austriaco, la cual ha sido abordada esencialmente en "Hans Kelsen un pensatore democratico tra Europa e America (1920-1955)". ¿Podría pintarnos un retrato del Hans Kelsen de este segundo periodo? ¿En qué momento marcha al exilio americano? ¿Cómo fue su llegada a los Estados Unidos de América? Una vez en la Unión Americana, ide qué manera desarrolla sus actividades docentes? ¿Cuáles son los intereses y preocupaciones de este Hans Kelsen más maduro?

SL: Kelsen llegó a América en 1940 para escapar, tanto de una Europa devastada por la guerra como de la Alemania nazi. Él nació en el seno de una familia judía, aunque después se convirtió, sobre todo por razones de conveniencia, a la religión cristiana; sin embargo, debido a la férrea ideología racista alemana, continuó siendo un enemigo. Ya en el nuevo mundo, su vida no fue inicialmente fácil. Solo después de algunas peregrinaciones, aterrizó finalmente en Berkeley, donde, en 1942, obtuvo la cátedra de Political Science. Este aspecto podría sorprender un poco. ¿Por qué ciencia política y no derecho? Porque, como ha sido oportunamente destacado por la literatura reciente, el Kelsen llegado a América es sustancialmente recibido con cierto recelo. En primer lugar, el formalismo jurídico no tenía seguidores en las universidades americanas. Por si fuera poco, el currículo de las facultades de derecho estadunidenses era menos abstracto y teórico que en las europeas. Kelsen, con su formación científica y académica, era, por tanto, percibido como no alineado totalmente con aquel tipo de ambiente científico y educativo. Desde el punto de vista de la teoría política, Kelsen no se encontró en una situación muy agradable; su concepción de la democracia era considerada muy abstracta y, en particular, su defensa del relativismo, en cuanto visión filosófica de la democracia, fue percibida como una expresión de una sustancial indiferencia hacia el tema de los valores. Empero, el periodo americano se reveló prolífico y rico en estímulos. Kelsen se dedicó a los estudios "internacionalistas", publicando obras como Peace through Law (1944), Will the judgement of the Nuremberg Trials constitute a precedent in international Law? (1947), The Law of the United Nations (1950). Se dedicó con pasión a la teoría del derecho internacional, y estudios recientes están reexaminando de manera crítica la definición de idealista que, con demasiada frecuencia, ha sido atribuida a su concepción del ordenamiento internacional y primacía del derecho internacional como instrumento de pacificación política. 
En América, Kelsen fue también consultor de la Comisión de las Naciones Unidas. Entre 1944 y 1955, junto a otros celebres emigrantes europeos, acabó bajo el objetivo del FBI, que estaba a la caza de posibles infiltrados comunistas en el territorio americano. Siempre me ha hecho sonreír este episodio de su vida americana; Kelsen fue, declarada y coherentemente, antimarxista y antisoviético durante toda su vida. Baste pensar en su Sozialismus und Staat (1923) o en su The Political Theory of Bolscevism (1948). Empero, la obsesión anticomunista americana había llegado al punto de sospechar inclusive de él, solo porque provenía de Europa y había expresado en algunas ocasiones aprecio por el programa socialdemócrata. Además, en Austria, Kelsen había estado vinculado por una gran amistad y estima al líder socialdemócrata Karl Renner. Muy probablemente, para sus interlocutores americanos era difícil entender que se podía simpatizar con la socialdemocracia y, al unísono, oponerse al marxismo. En cualquier caso, en América, el jurista, que ya no era joven, encontró refugio y la oportunidad de construirse una nueva existencia. Como prueba de que, evidentemente, en su nueva patria se encontraba bien, decidió pasar la última parte de su vida y murió en Berkeley en 1973.

AFCS: A decir verdad, usted no deja de sorprendernos. iEs una académica infatigable! iSu pasión por estudiar la vida y obra de Hans Kelsen es inagotable! Así, recientemente ha publicado un nuevo libro intitulado Democracy in its Essence: Hans Kelsen as a Political Thinker. ¿Cuáles son los temas centrales de esta interesantísima obra? ¿En qué forma surge el interés por escribir este nuevo libro?

SL: Es un libro pensado para un público americano (norteamericano y latinoamericano) que he escrito con una única y simple finalidad: ofrecer una introducción razonada a la teoría democrática de Kelsen, destacando cuatro grandes temas que, en mi opinión, caracterizan profundamente su reflexión sobre el significado de la democracia moderna, sea en el periodo europeo, sea en el periodo americano: pluralismo, constitucionalismo, relativismo y procedimentalismo. En un momento en que, en varias partes del mundo, la democracia representativa afronta retos increíbles, volver a hablar de un pensador que, en el momento más oscuro de la historia europea del siglo XX, percibió la necesidad de entender cuáles podrían ser algunos elementos fundamentales que distinguiesen el gobierno democrático de aquel autocrático (cualquiera que sea su orientación ideológica) podría ser de mucha utilidad. 
No porque necesariamente se deba estar de acuerdo con el punto de vista de Kelsen, sino porque es útil tratar de medirse con el pensamiento de quien se ha interrogado sobre la esencia y valor de la democracia en un periodo de crisis y ha tratado de defenderla frente a sus acérrimos detractores.

Mi perspectiva metodológica sigue siendo rigorosamente histórica, es decir, orientada a mostrar las conexiones entre su concepción política y el contexto histórico en que vivió y trabajó. He empleado un lenguaje accesible y he preferido la síntesis. Espero que esta elección pueda animar a la lectura del libro.

AFCS: Por desgracia, todo lo que tiene un inicio posee también un final. Me gustaría hacerle un par de preguntas conclusivas. ¿Qué caminos seguirán sus investigaciones en los siguientes años? ¿Sobre qué temas le gustaría escribir? Si tuviera que aconsejar a los futuros investigadores del pensamiento de Hans Kelsen, ¿qué senderos les sugeriría? ¿cuáles son las parcelas más fértiles, pero poco labradas, de la vida y pensamiento del maestro de Praga?

SL: Aún queda mucho por escribir y estudiar. La mía es, tan solo, una pequeña contribución. Yo misma -cada vez que vuelvo a reflexionar sobre su obra- tengo la impresión de que siempre hay algo que se me ha escapado o que se me escapa, y algunas consideraciones que había expresado hace unos años ahora no me parecen tan convincentes.

En general, en los últimos años, este pensador ha despertado un gran interés a nivel internacional. Ha habido algunos estudiosos que han identificado, en su teoría democrática, algunos posibles antídotos -en el plano conceptual-contra el populismo. Otros han estado reevaluando los aspectos más propiamente realistas de su doctrina internacionalista. Algunos más han estado indagando de manera más profunda su periodo americano, así como la relación entre su teoría jurídica y política. No creo que haya terrenos del todo inexplorados. Quizá amerita un estudio más profundo, por ejemplo, el vínculo entre Kelsen y Freud; empero, ya existen algunos trabajos interesantes al respecto que han aparecido recientemente. Por lo que a mí respecta, sin embargo, estaría muy interesada en indagar sobre la recepción de Kelsen en América Latina. Es un tema sobre el cual, en Europa, no se ha escrito mucho y, ciertamente, no porque no sea importante, sino más bien porque en ocasiones nosotros, del Viejo Continente, tendemos a ser un tanto autorreferenciales. Al despedirme, le agradezco esta entrevista, sus hermosas preguntas y espero, aunque sea en una mínima parte, haberle dado respuestas suficientemente completas e interesantes. 
